\title{
Cost Recommendation under Uncertainty in IQWiG's Efficiency Frontier Framework
}

\author{
Isaac Corro Ramos, PhD, Stefan K. Lhachimi, PhD, \\ Andreas Gerber-Grote, MD, PhD, Maiwenn J. Al, PhD
}

\begin{abstract}
Background. The National Institute for Quality and Efficiency in Health Care (IQWiG) employs an efficiency frontier (EF) framework to facilitate setting maximum reimbursable prices for new interventions. Probabilistic sensitivity analysis (PSA) is used when yes/no reimbursement decisions are sought based on a fixed threshold. In the IQWiG framework, an additional layer of complexity arises as the EF itself may vary its shape in each PSA iteration, and thus the willingness-to-pay, indicated by the EF segments, may vary. Objectives. To explore the practical problems arising when, within the EF approach, maximum reimbursable prices for new interventions are sought through PSA. Methods. When the EF is varied in a PSA, cost recommendations for new interventions may be determined by the mean or the median of the distances between each intervention's point estimate and each EF. Implications of using these metrics were explored in a simulation study based on the model used by IQWiG to assess the cost-effectiveness of 4 antidepressants. Results. Depending on the metric used,
\end{abstract}

cost recommendations can be contradictory. Recommendations based on the mean can also be inconsistent. Results (median) suggested that costs of duloxetine, venlafaxine, mirtazapine, and bupropion should be decreased by $€ 131, € 29$, €12, and €99, respectively. These recommendations were implemented and the analysis repeated. New results suggested keeping the costs as they were. The percentage of acceptable PSA outcomes increased $41 \%$ on average, and the uncertainty associated to the net health benefit was significantly reduced. Conclusions. The median of the distances between every intervention outcome and every EF is a good proxy for the cost recommendation that would be given should the EF be fixed. Adjusting costs according to the median increased the probability of acceptance and reduced the uncertainty around the net health benefit distribution, resulting in a reduced uncertainty for decision makers. Key words: detailed methodology: probabilistic sensitivity analysis; simulation methods; decision analysis. (Med Decis Making 2017;37:162-172)
I $n$ the German system of statutory health insurance-covering approximately $90 \%$ of the German population-the assessment of the benefits

Received 6 May 2015 from the Institute for Medical Technology Assessment, Erasmus University Rotterdam, the Netherlands (ICR, MJA); Research Group Evidence-Based Public Health, Leibniz-Institute for Epidemiology and Prevention Research (BIPS), Bremen, Germany (SKL); Health Sciences Bremen, Institute for Public Health and Nursing, University Bremen, Germany (SKL); and Institute for Quality and Efficiency in Health Care (IQWiG), Cologne, Germany (SKL, AG). Revision accepted for publication 2 February 2016.

Address correspondence to Isaac Corro Ramos, PhD, Institute for Medical Technology Assessment (iMTA) Erasmus University Rotterdam, PO Box 1738, Room J8-27, 3000 DR Rotterdam, the Netherlands; telephone: +31-10-4081829; fax: +31-10-4089081; e-mail: corroramos@ imta.eur.nl.

(c) The Author(s) 2016

Reprints and permission:

http://www.sagepub.com/journalsPermissions.nav

DOI: $10.1177 / 0272989 \times 16636856$ and costs of drugs was legislated as a remit for the National Institute for Quality and Efficiency in Health Care (IQWiG) in 2007 (see Social Code Book [SGB] V § 35b). To comply with the legislative stipulation, IQWiG published its general methods for the assessment of the relation between benefits and costs after extensive consultations in $2009 .{ }^{1}$ At the core, an efficiency frontier framework was introduced. In IQWiG's efficiency frontier approach, existing therapies (interventions) for a given health problem are plotted in a cost-effectiveness plane, with intervention costs per patient on the x-axis and outcomes (measured, for instance, as qualityof-life scores) on the y-axis. An example of this can be seen in Figure 1. The enveloping line and its extrapolation to the right is called the efficiency frontier. New interventions that locate themselves to the right and below the frontier may prima facie be judged as unduly costly relative to the benefits they provide. With such interventions, IQWiG seeks 


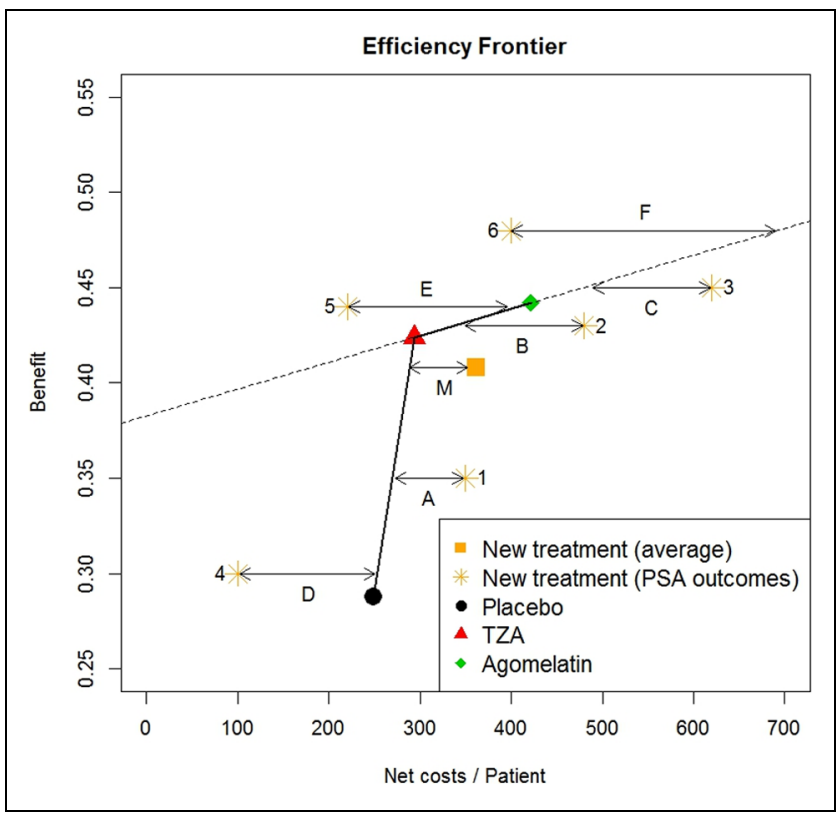

Figure 1 Probabilistic sensitivity analysis (PSA) outcomes and cost adjustment. TZA, tricyclic antidepressants.

price reductions such that the interventions move to the frontier. The approach assists in defining indication-specific willingness-to-pay thresholds, ${ }^{2}$ expressly avoiding fixed thresholds (for the legal and political reasons motivating the methodological choice, see Gerber-Grote et al. ${ }^{3}$ and Sandmann et al. ${ }^{4}$ and the references therein). The detailed setup of an efficiency frontier within the German ${ }^{5}$ and other settings ${ }^{6-14}$ has been described elsewhere. The efficiency frontier is also used in Belgium to select an appropriate comparator ${ }^{15}$ and in France to calculate incremental cost-effectiveness ratios for nondominated interventions. ${ }^{16}$ Even though the scope of these efficiency frontiers differs from the objective of IQWiG's, there are similar challenges (e.g., those concerning how to account for uncertainty in the efficiency frontier framework).

In November 2013, the first assessment of costs and effects of new drugs within one therapeutic area was published by IQWiG. This was the assessment of 4 drugs in the indication of depression. ${ }^{17}$ At the time when the commission was assigned in December 2009 by the Federal Joint Committee, venlafaxine, for instance, was considered a fairly new drug that had additional benefit over its comparators at comparatively high costs. For this article, the case is only of illustrative nature. It should be emphasized that the original efficiency frontier concept did not deal with uncertainty, but in the IQWiG assessment of depression, uncertainty was explicitly explored. While assessing these costs and effects through a health economic model (based on the methods guide $^{1}$ ), a potential methodological problem arose, relating to the analyses of uncertainty through a probabilistic sensitivity analysis (PSA). The PSA accounts for the uncertainty around input values for the model and leads to a set of possibly highly varying combinations of costs and effects on the cost-effectiveness plane. ${ }^{7,18}$ PSAs are commonly used when a yes/no reimbursement decision is sought based on a fixed threshold, like in the UK National Institute for Health and Care Excellence (NICE) setting.

Given the uncertainty around the estimates of costs and/or effects of existing therapies, the efficiency frontier is actually not known as a precisely deterministic line on the cost-effectiveness plane. Since the location of new interventions on the costeffectiveness plane is also uncertain, the horizontal distance between the new intervention and the efficiency frontier (i.e., the difference between actual and required cost per patient) will vary per PSA simulation. Thus, in the efficiency frontier framework, an additional layer of complexity arises compared with the fixed-threshold framework as the efficiency frontier itself is also probabilistic in practice, and the method used to address the problem when the efficiency frontier is deterministic does not directly apply in the probabilistic setting. Therefore, before IQWiG could formally move from a deterministic to a probabilistic approach (in case IQWiG decides to do this), this issue needs to be resolved; otherwise, there is no alternative approach for the current deterministic one. When this problem is solved, any views on whether decision making should rely on deterministic or probabilistic outcomes can be based on principles rather than practicalities. However, this article does not deal with this underlying question.

In this article, we first explain how cost recommendations for new interventions in the efficiency frontier framework should be determined when uncertainty around model outcomes is taken into consideration through a PSA. It is important to stress that cost recommendations are given for new interventions only, and such recommendations are given as an output of a PSA (i.e., after a PSA is run). In a PSA, the position of the cost and effect estimates of the treatments that form the efficiency frontier will vary on the cost-effectiveness plane due to the uncertainty around those estimates, but no further 
cost recommendation for the treatments that form the efficiency frontier will be given in any way. Then, we describe and present the results of a simulation study that was set up to illustrate the practical problems encountered when the efficiency frontier is probabilistic. This simulation is based on the model developed by a group of experts in cooperation with IQWiG to assess the cost-effectiveness of antidepressants. ${ }^{17}$ In a large number of PSA simulations, some results (i.e., the horizontal distance between the new intervention and the efficiency frontier) may be extreme. In that situation, the median is theoretically a better measure of central tendency than the mean. We demonstrate the difference between the performance of the two and the implications in cost recommendations within IQWiG's efficiency frontier framework.

\section{Cost Recommendation under Uncertainty in IQWiG's Efficiency Frontier}

Simply speaking, the main aim of the efficiency frontier is to determine an appropriate price for new interventions within the German system of statutory health insurance. When a new intervention is below the efficiency frontier, its cost must be reduced so that it becomes acceptable by bringing it exactly to the efficiency frontier. This implies that the location of the new intervention on the cost-effectiveness plane might only be modified horizontally-that is, the benefits are considered to be given, but the cost could be adjusted (in this case reduced), for example, by the intervention's manufacturer. (The cost reduction [or increase] mentioned here is not equal to the price reduction for a given intervention as reported in the cost-effectiveness evaluation of antidepressants by IQWiG. ${ }^{1}$ However, there is a [linear] relationship between the price of the new intervention and the total cost associated to it so that when the cost reduction is known, the price reduction should be easily calculated. Since this relationship was unknown to us, we present all the results in terms of cost instead of price.) This idea is illustrated in Figure 1, where the efficiency frontier is determined by placebo, tricyclic antidepressants (TZA [Trizyklische Antidepressiva in German]), and agomelatine, and the outcomes, labeled 1 to 6 , are the results of PSA iterations on the new intervention. (All tricyclic antidepressants plus maprotiline were considered as a drug class in the preceding benefit assessments performed by IQWiG. ${ }^{19,20}$ If no heterogeneity could be detected for these drug classes, lead compounds were determined as representatives of drug classes.)
The outcomes for the new intervention, labeled 1, 2 , and 3 , are not accepted unless their costs are reduced by $€ 77$ (A), €131 (B), and $€ 130$ (C), respectively. Note that different segments of the efficiency frontier are used to determine the cost reduction. Likewise, when a new intervention is located above the efficiency frontier, its cost is considered appropriate. However, given its level of benefits, a higher cost (determined by its horizontal distance to the efficiency frontier) may also be acceptable. For example, the cost of the outcomes for the new intervention, labeled 4, 5, and 6 in Figure 1, could be increased by $€ 150$ (D), $€ 175$ (E), and $€ 290$ (F), respectively, and still be considered appropriate. Note finally that the cost of a new intervention does not need to change if it lies on a segment of the efficiency frontier, since the tradeoff between costs and benefits, as stipulated by this segment, is in the IQWiG context often considered a willingness-to-pay in a given field of indication.

When a PSA is now performed, not only the point estimate of the new intervention changes but also those of all comparators on the cost-effectiveness plane, resulting in as many different efficiency frontiers as PSA iterations. Depending on its position relative to the efficiency frontier, a cost reduction or increase for the new intervention may be considered.

For didactic reasons, we keep the efficiency frontier fixed in a first series of PSA iterations where only the position of the new intervention changes. It is important to emphasize that the distance between the average point estimate of the new intervention and the efficiency frontier (further referred to as metric M1) is not necessarily the same as the average of the distances between every single PSA outcome of the new intervention and the fixed efficiency frontier. Furthermore, if the latter is used to determine a cost reduction or increase, it may lead to erroneous decisions. We will explain this with the help of Figure 1. As indicated above, we assume a fixed efficiency frontier, while the outcomes labeled 1 to 6 are the results of PSA iterations on the new intervention. The average of the 6 point estimates of the new intervention (solid square) lies below the efficiency frontier, and thus the new intervention would be rejected and the horizontal distance $M$ suggests a $€ 75$ cost reduction. However, when computing all the individual distances between the PSA outcomes 1 to 6 and the efficiency frontier, we get cost reductions A $(€ 77)$, B $(€ 131)$, and $C(€ 130)$ and cost increases D (€150), E (€175), and $F(€ 290)$. On average, this would suggest a $€ 46$ cost increase, which contradicts the $€ 75$ cost 
Table 1 Simulation Assumptions

\begin{abstract}
Assumptions
1. The new health care intervention does not need to lower the efficiency of the relevant therapeutic area. Thus, any possible outcomes for the new intervention were allowed on the cost-effectiveness plane so that all the areas below and above the efficiency frontier were used.

2. We have considered a general situation where the first intervention included on the efficiency frontier (the one with the lowest costs and benefits) was not placed at $(0,0)$ on the cost-effectiveness plane.

3. We have assumed that the costs of the new intervention were always positive and the benefits were constrained between zero and 1.

4. We considered placebo as "doing nothing." Thus, when the first intervention included on the efficiency frontier was placebo, every value of a new intervention providing less benefit than placebo was considered unacceptable (i.e., it would be unethical to "do something" as opposed to "do nothing" so that the patient loses health, even if it leads to cost saving).

5. We assumed that there are at least 2 interventions determining the efficiency frontier. Thus, we did not consider the situation where only 1 intervention could be plotted.
\end{abstract}

reduction suggested by the distance $M$. A cost increase would keep the average point estimate of the new intervention below the efficiency frontier and may decrease the probability of accepting the new intervention since some of the outcomes located to the left of the efficiency frontier may move to the right of the efficiency frontier and become rejected.

It should be emphasized that to appropriately represent uncertainty within the efficiency frontier, 2 distinctive yet simultaneously occurring aspects of uncertainty must be accounted for in each iteration of a PSA. First, the new intervention may vary its position on the cost-effectiveness plane (as shown in Figure 1), and second, all other treatment strategies may vary their position on the cost-effectiveness plane as well. Because of the latter, the efficiency frontier may vary its shape, and crucially, the tradeoff between costs and effects (willingness-to-pay) as indicated by the segments of the efficiency frontier may vary between each iteration of the PSA. Therefore, in the case of a probabilistic efficiency frontier, metric M1 cannot be computed. We can only compute the distance from every PSA outcome of the new intervention and the efficiency frontier generated in this particular PSA iteration. The average of these individual distances will be referred to as metric M2. A practical problem now is that the distribution of the individual distances is skewed (and may have extreme values). Because of that, if price recommendations were based on M2, it may result in a biased estimation of the real recommended price. In cases of skewed distributions with heavy tails, statistical theory suggests that a median (here of the individual distances; further referred to as metric M3) is a better statistic than M2 to represent the typical value of the distance from a PSA outcome of the new intervention to the PSA outcome of the efficiency frontier. ${ }^{21}$ This issue was studied with a simulation study based on the model developed by IQWiG to assess the cost-effectiveness of antidepressants. ${ }^{17}$

\section{METHODS}

We set up a simulation study to assess cost recommendations based on PSA that was based on the model developed by IQWiG to assess the costeffectiveness of antidepressants. ${ }^{17}$ The assumptions we made in our simulations analyses are summarized in Table 1.

We used as a data set the results of the PSA described in the cost-effectiveness evaluation of antidepressants by IQWiG, ${ }^{17}$ which is the result of 100,000 PSA iterations. An example of the first 4 configurations of the cost-effectiveness plane can be seen in Figure 2. In each iteration, a new efficiency frontier and a point estimate (costs and benefits) of 4 new interventions (i.e., venlafaxine, duloxetine, bupropion, and mirtazapine) were generated. Therefore, the average point estimate for costs and benefits and the cost increase or reduction needed to bring that average to the efficiency frontier could not be computed here as there is no single (deterministic) efficiency frontier. Hence, we computed the average and the median of the individual distances between each intervention point estimate and each efficiency frontier (metrics M2 and M3, respectively) and studied the implications when cost recommendations would be based on each of these 2 metrics.

\section{RESULTS}

In all PSA iterations, placebo was the first element on the efficiency frontier (cheapest and with lowest 




Figure 2 Probabilistic sensitivity analysis (PSA) samples with probabilistic efficiency frontier. TZA, tricyclic antidepressants.

benefits). Most of the PSA iterations resulted in an efficiency frontier with $2(75 \%)$ and $3(21.64 \%)$ interventions. The most common efficiency frontiers were placebo-TZA and placebo-TZA-agomelatine. Details on the number of interventions per PSA iteration and the position of each intervention on the efficiency frontier can be seen in Table 2, and further descriptive statistics are shown in Table 3. The maximum willingness-to-pay threshold, which is determined by the last program on the efficiency frontier, is computed as 1 divided by the slope of the last segment on the efficiency frontier. Thus, the average of these 100,000 estimates of the maximum willingness-topay was equal to $€ 4.1 * 10^{13}$, whereas the median was $€ 237$ (cf. Table 3 ). Note that this average is extremely high due to the PSA iterations where the slope of the last segment was nearly zero. However, 
Table 2 Number of Interventions and Their Position on the Efficiency Frontier

\begin{tabular}{lclrrrr}
\hline \hline \multirow{2}{*}{$\begin{array}{l}\text { No. of Interventions on } \\
\text { the Efficiency Frontier }\end{array}$} & No. of PSA Iterations & Intervention & \multicolumn{3}{c}{ Position on the Efficiency Frontier } \\
\cline { 3 - 7 } 1 & 0 & Flacebo & 100,000 & 0 & 0 & 0 \\
2 & 75,040 & TZA & 0 & 94,913 & 4185 & 0 \\
3 & 21,645 & SSRI minimum & 0 & 5086 & 0 & 0 \\
4 & 3276 & Trazodone & 0 & 1 & 228 & 63 \\
5 & 39 & Agomelatine & 0 & 0 & 20,498 & 3252 \\
6 & 0 & SSRI maximum & 0 & 0 & 49 \\
\hline
\end{tabular}

PSA, probabilistic sensitivity analysis; SSRI, selective serotonin reuptake inhibitor; TZA, tricyclic antidepressants.

Table 3 Descriptive Statistics for the Slope of the Segments of the Efficiency Frontier and the Associated Willingness-to-Pay

\begin{tabular}{lccccccc}
\hline \hline & $\boldsymbol{n}$ & Average & Median & 2.5th Percentile & 97.5th Percentile & Minimum & Maximum \\
\hline Slope last segment & 100,000 & 0.0035 & 0.0042 & 0.00002 & 0.0064 & $3^{*} 10^{-18}$ & 0.0091 \\
Willingness-to-pay $(\lambda), €$ & 100,000 & $4.1^{*} 10^{13}$ & 237 & 156 & 40,022 & 109 \\
Slope segment 1 & 100,000 & 0.0043 & 0.0043 & 0.0025 & 0.0064 & 0.0007 \\
Willingness-to-pay 1, $€$ & 100,000 & 242 & 231 & 155 & 395 & 0.0091 \\
Slope segment 2 & 24,960 & 0.0004 & 0.0001 & 0.000006 & 0.0028 & 109 & 0 \\
Willingness-to-pay 2, $€$ & 24,960 & 35,527 & 5713 & 344 & 146,634 & 0.0042 \\
Slope segment 3 & 3315 & 0.0002 & 0.0002 & 0.00001 & 0.0006 & 0.0000003 & 0.0016 \\
Willingness-to-pay 3, $€$ & 3315 & 13,817 & 4136 & 1440 & 76,630 & 607 & $2,692,600$ \\
Slope segment 4 & 39 & 0.0001 & 0.0001 & 0.00002 & 0.0005 & 0.00001 & 0.0005 \\
Willingness-to-pay 4, $€$ & 39 & 13,700 & 6795 & 1950 & 47,567 & 1813 & 74,956 \\
\hline
\end{tabular}

Table 4 Percentage of Acceptance and Cost Recommendations Based on Metrics M2 and M3

\begin{tabular}{|c|c|c|c|c|c|c|}
\hline & \multicolumn{3}{|c|}{ Before Implementing Cost Recommendation } & \multicolumn{3}{|c|}{ After Implementing Cost Recommendation (Based on the Median M3) } \\
\hline & \multirow[b]{2}{*}{ Acceptance, $\%$} & \multicolumn{2}{|c|}{ Cost Recommendation, $€$} & \multirow[b]{2}{*}{ Acceptance, $\%$} & \multicolumn{2}{|c|}{ Cost Recommendation, $€$} \\
\hline & & M2 & M3 & & M2 & M3 \\
\hline Duloxetine & 0.28 & -128 & -131 & 49.65 & 3 & 0 \\
\hline Venlafaxine & 13.92 & 33 & -29 & 48.67 & 62 & 0 \\
\hline Mirtazapine & 12.22 & 1 & -12 & 47.96 & 13 & 0 \\
\hline Bupropion & 0.10 & -99 & -99 & 43.81 & 0 & -1 \\
\hline
\end{tabular}

A negative monetary value means cost reduction, whereas a positive one means cost increase. M2, average of the cost recommendations based on the probabilistic sensitivity analysis (PSA) outcomes separately; M3, median of the cost recommendations based on the PSA outcomes separately.

*Simulations providing an infinite cost increase were excluded from the computation of the average ( 16 for duloxetine, 49 for venlafaxine, 30 for mirtazapine and 2 for bupropion).

this average does not correspond to the standard definition of a probabilistic incremental cost-effectiveness ratio (ICER) as $\overline{\Delta C} / \overline{\Delta E}$, where $\overline{\Delta C}$ and $\overline{\Delta E}$ denote the average incremental costs and effects, respectively, which should be closer to the median.

Results regarding acceptance and cost recommendations have been summarized in Table 4. For all the new interventions, the percentage of acceptable PSA outcomes (above the efficiency frontier) was low, with $13.92 \%$ for venlafaxine being the highest. Mirtazapine had a similar percentage of acceptance $(12.22 \%)$, but for duloxetine and bupropion, these were almost zero.

In the original data set, the average cost of duloxetine, venlafaxine, mirtazapine, and bupropion was $€ 408$, €319, €295, and €372, respectively. Depending 
on the metric used, cost recommendations based on M2 and M3 can be contradictory, as occurred here, especially with venlafaxine and to a lower extent with mirtazapine. This is because the distribution of the individual distances between PSA outcomes of the new intervention and the PSA efficiency frontiers is most asymmetric for venlafaxine. In that case, recommending a cost increase for both venlafaxine $(€ 33)$ and mirtazapine $(€ 1)$ does not seem appropriate since this, for example, would decrease the percentage of acceptable PSA outcomes. Moreover, if this cost increase was implemented and M2 recalculated, the updated recommendation based on M2 would result in a cost decrease since the recalculated M2 would be negative. Based on the average M2, the cost of duloxetine and bupropion should be decreased by $€ 128$ and $€ 99$, which are similar to those recommended by the median M3. However, the simulations providing a (numerically) infinite distance (or equivalently an infinite cost increase) were excluded from the computation of the average M2, and therefore the cost recommendation based on M2 shown in Table 4 is likely to be an underestimation. Based on the median of the distances between every PSA outcome and every efficiency frontier (M3), these simulations are also included, and as explained above, the median is more representative of the typical observation than the average, and it is the preferred measure of central tendency of the population under study. Thus, based on the simulation results, the cost of duloxetine, venlafaxine, mirtazapine, and bupropion should be decreased by $€ 131$, $€ 29$, €12, and €99, respectively, as recommended by the median M3.

We implemented the cost recommendations based on the median and repeated the analysis. An example of the first 4 configurations after implementing the cost recommendations can be seen in Figure 3. We can observe that compared with Figure 2, the position of the new interventions has been simply shifted toward the efficiency frontier.

The results after the cost recommendations based on the median were implemented (see Table 4) showed that the percentage of acceptable PSA outcomes increased significantly, with $43.81 \%$ for bupropion being the lowest and $49.65 \%$ for duloxetine being the highest. The average cost of duloxetine, venlafaxine, mirtazapine, and bupropion was now $€ 277$, $€ 290$, $€ 283$, and $€ 273$, respectively (i.e., the cost before recommendation minus the recommended reduction). Updated recommendations based on the median (M3), as expected, were $€_{0}$ (except for bupropion, which was $-€ 1$, but this may be caused by rounding up the costs). Note finally that if updated recommendations were based on the average M2, this would imply a cost increase for all interventions (except for bupropion), which would imply returning to the situation before update, which is clearly worse than the situation after implementing cost recommendations based on the median.

Moreover, we have also observed that the uncertainty associated with the net health benefit (NHB) has been reduced after implementing the cost recommendation, as can be seen in Figure 4. Note that the NHB was graphically defined as the vertical distance between a point estimate and the last segment (or its extension-backward or forward) on the efficiency frontier. ${ }^{5}$ Thus, it is not only that there are many more PSA outcomes above the efficiency frontier but also that the PSA outcomes are closer to the last segment on the efficiency frontier (for that reason, we observed that the distribution of the NHB after implementing the cost recommendations is centered at zero). Since in most of the cases, the efficiency frontier had exactly one segment (around 75\%), it also means that the PSA outcomes are in fact closer to the efficiency frontier. As can be seen in Figure 4, most of the PSA outcomes for the new intervention were originally located to the right of the efficiency frontier, where the NHB is negative. If the original distribution of the PSA outcomes had laid more across the frontier, the effect of implementing the cost recommendation on the NHB distribution would have been smaller.

\section{DISCUSSION}

In this article, we have explored the potential problems and some solutions arising when, within the IQWiG efficiency frontier approach, a maximum reimbursable price for a new intervention is sought through probabilistic sensitivity analyses. Cost recommendations on the efficiency frontier seem to be prone to skewed distributions. In these situations, statistical theory says that the median is a better measure of central tendency than the mean, and for price negotiations within a probabilistic efficiency frontier framework, this should be noted. To our knowledge, this issue was never raised in the field of health economics.

We have first explained that when the efficiency frontier is deterministic, cost recommendations should be based on the average point estimate of the PSA outcomes of the new intervention. An increase 


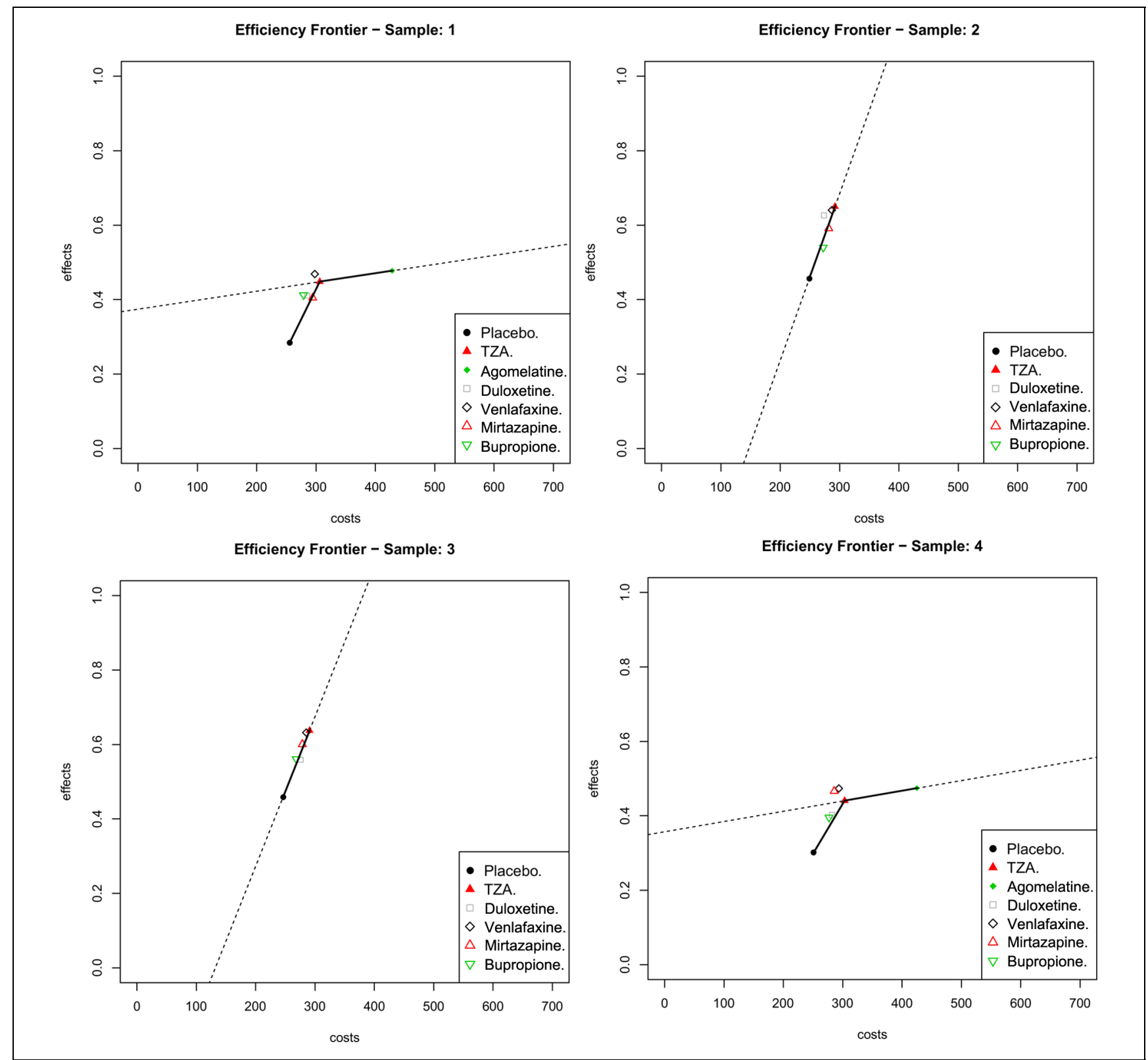

Figure 3 Probabilistic sensitivity analysis (PSA) samples with probabilistic efficiency frontier after implementing cost recommendations. TZA, tricyclic antidepressants.

or decrease in cost recommendation is then calculated as the horizontal distance between the average point estimate and the efficiency frontier (metric M1). In general, this metric is not the same as the average of the distances between every PSA outcome and the efficiency frontier (metric M2). In fact, we have observed that metric M2 was always larger than metric M1. This is because the distribution of metric M2 was affected by very large values.
When the efficiency frontier is probabilistic (i.e., it alters its shape in each PSA iteration), cost recommendations cannot be based on the distance between the average point estimate of the PSA outcomes and the efficiency frontier (metric M1) since this cannot be computed. Based on the results obtained when the efficiency frontier is deterministic (results not shown), we expected that the median M3 should be preferred over the average M2 when the efficiency 

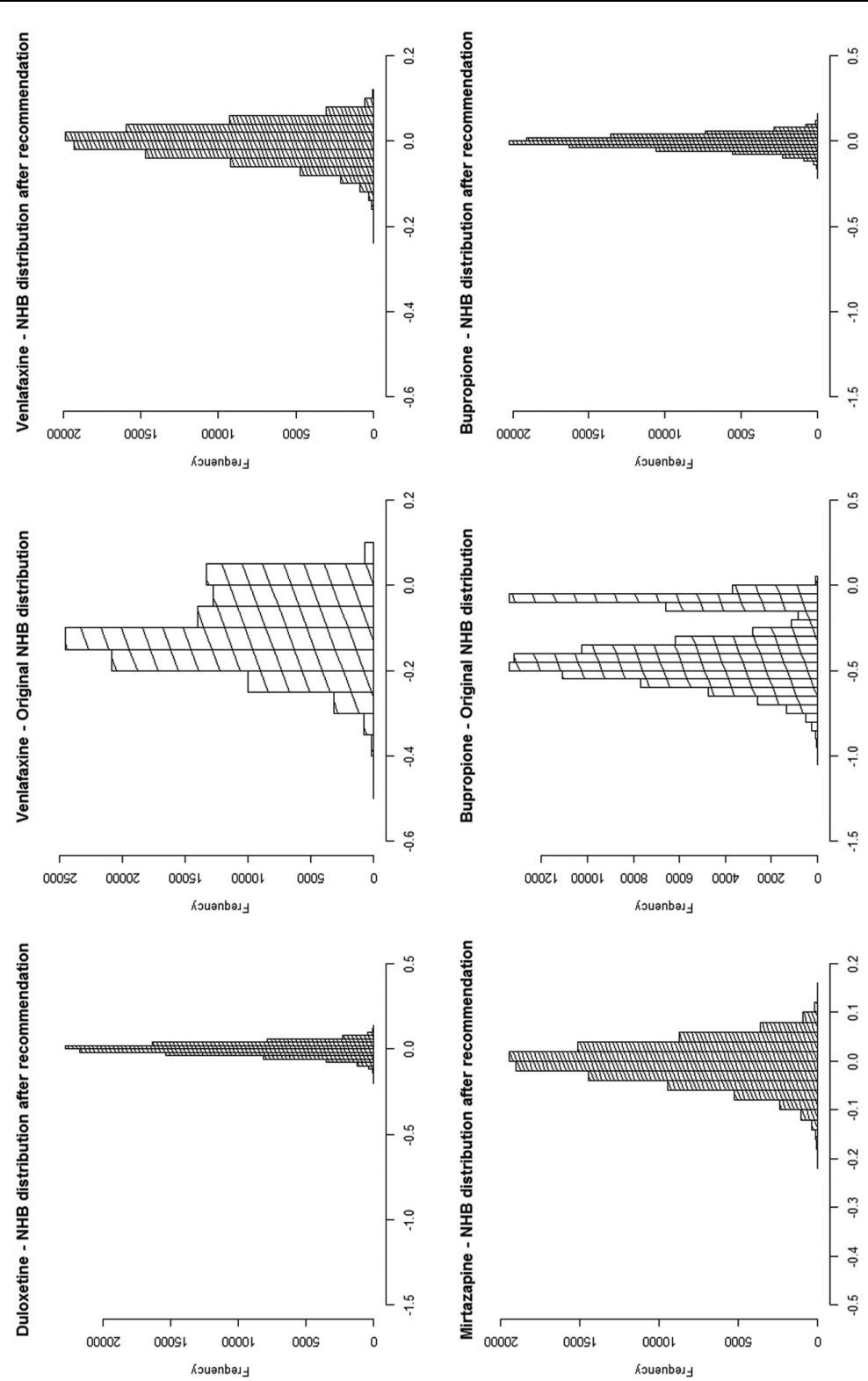

हี

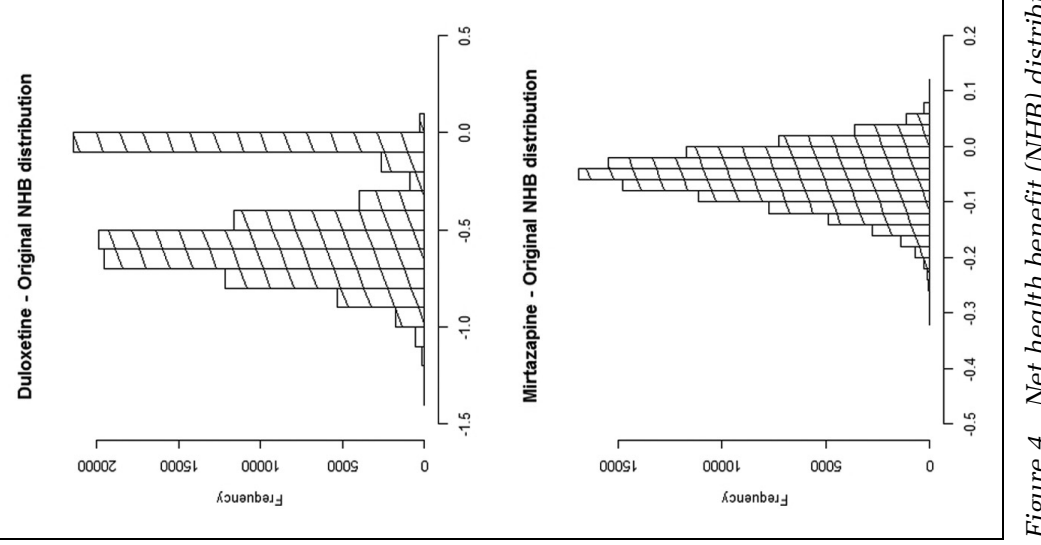


frontier is probabilistic. In this situation, theory confirms that the median (metric M3) is more representative of the typical observation than the average (metric M2), and it is the preferred measure of central tendency of the population under study (see, e.g., Ross, ${ }^{21}$ Section 3.3). With our simulation analysis, we could demonstrate that also in this case, the median M3 is in general better than M2. Moreover, when calculating the distances between every PSA outcome and every efficiency frontier, we found that for all the new interventions, some simulations provided a (numerically) infinite distance (or equivalently a numerically infinite cost increase). Note that this is due to the PSA iterations where the slope of the last segment was nearly zero. This also has implications in the maximum willingness-to-pay threshold (which is computed as 1 divided by the slope of the last segment on the efficiency frontier) and therefore in measures like the net health or monetary benefit, since the average willingness-to-pay can be (numerically) infinite. In this case, the median also provides a better estimate, as shown in Table 3. Descriptive statistics for each segment separately are also presented in Table 3. That way, we can observe what the contribution of each segment to the maximum willingnessto-pay might be. This illustrates that, as expected, the distribution of the cost recommendations based on the PSA outcomes separately can be highly skewed and affected by the presence of extreme values. Note that such extreme values are not statistical outliers in a formal sense. An outlier can be defined as an observation that is very different from other observations in a set of collected data. Given a skewed distribution with heavy tails and/or a rather small sample size, a single outlier may heavily affect the sample. In our simulation, we do have simulated model outcomes that heavily affect the mean of the distances as they resulted in infinite distances. We have seen that this happens often in the PSA, even though the PSA size was very large $(10,000)$. Therefore, we may assume that the median (metric M3) instead of the average (metric M2) should be used to determine price recommendations in this setting. The average (metric M2) would overestimate the cost recommendation, which can be very wrong. The median (metric M3) is less sensitive to skewed distributions and extreme values, and when the efficiency frontier is probabilistic, cost recommendations should be based on it.

Our analysis has also confirmed that the median M3 is a good proxy for the cost recommendation that would be given should the efficiency frontier be fixed. After implementing the cost recommendations based on the median, we have seen that the "updated" cost recommendation was $€ 0$, but also the probability of acceptance increased substantially, which would not occur if M2 would be used to recommend the costs. Note that the percentage of acceptable PSA outcomes is expected to be close to $50 \%$ as a consequence of the cost being reduced by the median. The fact that the percentage is not exactly $50 \%$ in all cases is due to the efficiency frontier having more than one segment in some of the PSA iterations. On top of that, the NHB distribution became narrower, which would result in a reduced uncertainty for the decision maker.

IQWiG's general methods in its current form ${ }^{22}$ do not go into the question addressed in this article. Applying the median of the distances between each intervention point estimate and each efficiency frontier after PSA will be incorporated as a specification in the next version of IQWiG's methods. Prima facie, Belgium and France will not run into this problem as they do not use the efficiency frontier to determine prices. However, even if one wants to simply decide which of 2 comparators dominates another and thus forms the efficiency frontier, one might encounter the problem in a PSA if the mean and the median lies on 2 sides of the efficiency frontier. Therefore, this simulation is of assistance to anyone using the efficiency frontier.

The choice of the probability distributions for the input parameters in health economic models is a crucial step when performing a PSA. However, since PSA is a well-established method, in this article, we are not explaining how to perform it properly (see, e.g., Briggs ${ }^{7}$ ). In the current study, we used the simulated costs and benefits for all treatments, implicitly assuming that the PSA that generated these outcomes was performed in a standard and proper way. Details about how the PSA was performed can be found in the cost-effectiveness evaluation of antidepressants by IQWiG (Table 81). ${ }^{17}$ Thus, the way the PSA outputs are generated is not related to the choice between the mean and the median of the distances as a way to determine cost recommendations but to the validity of the estimates. Obviously, if the PSA is not performed correctly, the median and the mean of the distances would not be reliable estimates, but nevertheless, even in that case, we would always choose the median of the distances over the mean.

Finally, note that we had always at least one segment in the efficiency frontier to determine the needed or allowed costs. This will not generally be the case, as can be observed in the cost-effectiveness evaluation of antidepressants performed by IQWiG. ${ }^{17}$ Here, various situations (depending on scenario and 
outcome used) occur where placebo dominates all current intervention options. In such situation, there is no clear decision rule to state which outcomes are acceptable, as there is no revealed willingness-topay. An overarching question in this context, but beyond the scope of this article, is how to determine that a number of therapies are sufficient in a given area to provide a useful frontier. ${ }^{2}$ Hence, how to deal with those scenarios should be the subject of further discussion.

\section{ACKNOWLEDGMENTS}

We thank Gaby Srozcynski, Uwe Siebert, Eva Esteban, and Annette Conrads-Frank who did the modeling in the costeffectiveness analysis of antidepressants for IQWiG.

\section{REFERENCES}

1. IQWiG. General methods for the assessment of the relation of benefits to costs. Version 1.0. 2009. Available from: https:// www.iqwig.de/download/General_Methods_for_the_Assessment_ of_the_Relation_of_Benefits_to_Costs.pdf

2. Caro JJ, Nord E, Siebert U, et al. The efficiency frontier approach to economic evaluation of health-care interventions. Health Econ. 2010;19(10):1117-27.

3. Gerber-Grote A, Sandmann FG, Zhou M, et al. Decision making in Germany: is health economic evaluation as a supporting tool a sleeping beauty? Z Evid Fortbild Qual Gesundheitswes. 2014; 108(7):390-6.

4. Sandmann F, Gerber-Grote A, Lhachimi S. Factors that led to the implementation of the efficiency frontier approach in health economic evaluation in Germany: do not avoid the elephant in the room. Comment on Klingler et al. (Health Policy 109 (2013) 270-280). Health Pol. 2013;112(3):297-8.

5. Stollenwerk B, Lhachimi SK, Briggs A, et al. Communicating the parameter uncertainty in the IQWiG efficiency frontier to decision-makers. Health Econ. 2015;24(4):481-90.

6. Karlsson G, Johannesson M. The decision rules of cost-effectiveness analysis. Pharmacoeconomics. 1996;9(2):113-20.

7. Briggs AH. Handling uncertainty in cost-effectiveness models. Pharmacoeconomics. 2000;17(5):479-500.

8. Goeree R, O’Brien BJ, Blackhouse G, Marshall J, Briggs A, Lad R. Cost-effectiveness and cost-utility of long-term management strategies for heartburn. Value Health. 2002;5(4):312-28.

9. Murray CJ, Lauer JA, Hutubessy RC, et al. Effectiveness and costs of interventions to lower systolic blood pressure and cholesterol: a global and regional analysis on reduction of cardiovascular-disease risk. Lancet. 2003;361(9359):717-25.
10. Niessen LW, Dijkstra R, Hutubessy R, Rutten GE, Casparie AF. Lifetime health effects and costs of diabetes intervention. Neth J Med. 2003;61(11):355-64.

11. Rojnik K, Naversnik K, Mateović-Rojnik T, Primiczakelj M. Probabilistic cost-effectiveness modeling of different breast cancer screening policies in Slovenia. Value Health. 2008;11(2): 139-48.

12. Eckermann S, Briggs A, Willan AR. Health intervention assessment in the cost-disutility plane. Med Decis Making. 2008;28(2): 172-81.

13. Barton GR, Briggs AH, Fenwick EA. Optimal cost-effectiveness decisions: the role of the cost-effectiveness acceptability curve (CEAC), the cost-effectiveness acceptability frontier (CEAF), and the expected value of perfection information (EVPI). Value Health. 2008;11(5):886-97.

14. Hallinen TA, Soini EJ, Eklund K, Puolakka K. Cost-utility of different intervention strategies after the failure of tumour necrosis factor inhibitor in rheumatoid arthritis in the Finnish setting. Rheumatology (Oxford). 2010;49(4):767-77.

15. Cleemput I, Neyt M, Van de Sande S, Thiry N. Belgian Guidelines for Economic Evaluations and Budget Impact Analyses: Second Edition. Health Technology Assessment (HTA). Brussels: Belgian Health Care Knowledge Centre (KCE); 2012. KCE Report 183C. D/2012/10.273/54

16. Haute Autorité de Santé (HAS). Choices in methods for economic evaluation. 2011. Available from: http://www.has-san te.fr/portail/jcms/r_1499251/en/choices-in-methods-for-economicevaluation

17. IQWiG. Vorbericht G09-01. Kosten-Nutzen-Bewertung von Venlafaxin, Duloxetin, Bupropion und Mirtazapin im Vergleich zu weiteren verordnungsfähigen medika-mentösen Behandlungen. 2012. Available from: https://www.iqwig.de/download/ G09-01_Vorbericht_Kosten-Nutzen-Bewertung-von-VenlafaxinDuloxetin-Bupropion-und-Mirtazapin.pdf

18. Briggs AH. Probabilistic analysis of cost-effectiveness models: statistical representation of parameter uncertainty. Value Health. 2005;8(1):1-2.

19. IQWiG. Abschlussbericht A05-20C. Bupropion, Mirtazapin und Reboxetin bei der Behandlung der Depression. 2009. Available from: https://www.iqwig.de/download/A05-20C_Abschluss bericht_1-1_Bupropion_Mirtazapin_und_Reboxetin_bei_Depressi onen.pdf

20. IQWiG. Abschlussbericht A05-20A. Selektive Serotonin- und Noradrenalin-Wiederaufnahmehemmer (SNRI) bei Patienten mit Depressionen. 2009. Available from: https://www.iqwig.de/down load/A05-20A_Abschlussbericht_SNRI_bei_Patienten_mit_Depress ionen_V1-1.pdf

21. Ross SM. Introductory Statistics. Elsevier Science; 2010. Boston, MA.

22. IQWiG. Allgemeine Methoden Version 4.2. 2015. Available from: https://www.iqwig.de/download/IQWiG_Methoden_Version_ 4-2.pdf 